\title{
Analysis of Effectiveness of Apriori Algorithm in Medical Billing Data Mining ${ }^{\prime}$
}

\author{
Umair Abdullah \\ FUIEMS \\ Foundation University \\ Islamabad, PAKISTAN \\ umair_pitafi@yahoo.com
}

\author{
Jamil Ahmad \\ IQRA University, \\ Islamabad Campus, PAKISTAN. \\ jamil@iqraisb.edu.pk
}

\author{
Aftab Ahmed \\ FUIEMS \\ Foundation University, \\ Islamabad, PAKISTAN \\ aftab_ff@hotmail.com
}

\begin{abstract}
Apriori algorithm has been successfully used for finding the frequent item sets in retail data. This research is about finding associations between diagnosis and treatments. It has shown that the algorithm is equally beneficent for finding the large item sets and thus generating the association rules in medical billing data. Resemblance between medical bill and purchase bill is the motivation of using Apriori algorithm in this research work. Healthcare is a data rich domain. Medical practices, insurance companies and other health related organizations have collected huge volumes of data, thus attracting data mining researchers to explore it and find something beneficent from it.
\end{abstract}

Keywords: Data mining, Association rules, Medical billing

\section{I.Background}

Association rule mining, one of the most famous techniques of data mining, was first introduced in [1]. It aims to extract associations among sets of items in the transaction databases or other data repositories. The formal statement of association rule mining problem was first stated in [1] by Agrawal et al. Let $\mathrm{I}=\{\mathrm{I} 1, \mathrm{I} 2, \cdots, \mathrm{Im}\}$ be a set of $\mathrm{m}$ distinct items; $\mathrm{T}$ be a transaction that contains a set of items. $\mathrm{D}$ is a database with different transaction records. An association rule is an implication in the form of $X \rightarrow Y$, where $X, Y$ are sets of items called itemsets. $\mathrm{X}$ is called antecedent while $\mathrm{Y}$ is called consequent. The rule means $\mathrm{X}$ implies $\mathrm{Y}$. There are two important basic measures for association rules; support ( ) denoted as s and confidence ( ) denoted as c. Support ( ) of an association rule is defined as the percentage/fraction of records that contain $\mathrm{X}$ and $\mathrm{Y}$ to the total number of records in the database. The count for each item is increased by 1 every time the item is encountered in different transaction of database D during the scanning process. It means the support count does not take the quantity of the item into account. Confidence of an association rule is defined as the percentage/fraction of the number of transactions that contain $\mathrm{X}$ and $\mathrm{Y}$ to the total number of records that contain $X$. If the percentage exceeds the predefined threshold of confidence then $X \rightarrow Y$ is an interesting association rule. Confidence is a measure of strength of the association rules. Association rule mining is to find out association rules that satisfy the pre-defined minimum support and confidence from a given database [2]. The problem is usually decomposed into two sub-problems. One is to find the itemsets whose occurrences exceed a predefined threshold in the database; the itemsets are called frequent or large itemsets.
The second problem is to generate association rules from the large itemsets with the constraints of minimal confidence.

\section{A. Healthcare IT (HIT)}

Application of information technology in Healthcare domain has emerged as a new multi-million dollar industry. Huge investments being made by many government, and private organizations to produce health related tools. EMR (Electronic Medical Record) also known as EHR (Electronic Health Record) is one of the major healthcare IT software, being used by medical providers. Similarly a lot of revenue being generated by those IT organizations, which are related to healthcare. Top company of 2003 "GE Healthcare" founded in 2000 has annual revenue of $\$ 2.7$ billion. And the 100th company "MED 3000 Inc." (Founded in 1985) has \$7.3 million annual revenue [3].

\section{II.Adaptation of Apriori Algorithm}

Apriori algorithm famous for finding frequent item sets, initially used in sales - purchase domain, now being tested and utilized in various domains. This basic algorithm has proved to be the starting point of all association rules mining algorithms.

\section{A. Apriori Algorithm}

Apriori algorithm based on a priori property of data i.e. all the subsets of a frequent itemset are always frequent. So when a k-itemset is not frequent, it is not used for generating candidates of $\mathrm{k}+1$-itemsets. Basically there are two parts of this rule generation algorithm. At the end of first part, frequent itemsets (i.e. having support greater than minimum support) of maximum size are returned. Items in each itemset are assumed to be lexically sorted. Then a simple approach is used i.e. to generate rules from these frequent itemsets by finding there all subsets.

\section{B. Modifications in Apriori Algorithm}

In order to meet the requirements two main modifications have been done in Apriori algorithm in this research work, one modification in main Apriori algorithm and other modification in rule generation algorithm. First of all an additional argument $\mathrm{G}$, which is a set of items given by the user as antecedent, is passed to the algorithm. In candidate generation those itemsets are kept as frequent itemsets which are being closed i.e. not being able to combine with any other k-itemset. So these closed

This work is supported by Higher Education Commission (HEC) of Pakistan under 5000 Indigenous Scholarship Scheme 
itemsets are kept in all iteration of finding the frequent itemsets. At the end first phase results in frequent itemsets (have support greater than minimum support) of unequal length are returned. Then in the second phase instead of considering all the subsets, of each frequent itemset, as antecedent only the given $\mathrm{G}$ is considered as antecedent and F$\mathrm{G}$ as consequent (where $\mathrm{F}$ is a frequent itemset). Rule is saved if it qualifies the minimum support and minimum confidence criteria. Modified Apriori algorithm is shown below as lisp version of Apriori-modified algorithm.

\section{Prototype Software}

For the research purpose, prototype software has been developed in Allegro CL 3.0.2 version of Lisp. A total of approximately 75000 records loaded with this software. Apriori and Apriori-modified algorithm applied on total 16487 records of claims and charges combined. All this data is stored in the form of text files and loaded in the memory when software runs. Requirement of data in the memory is one of the weaknesses of Apriori algorithm which has not been overcome in this research work. All the internal memory management of the data structure has been done by Lisp itself.

Front end of the software, consists of a main starting window. Rule generation feature is provided on claim window, which is also used for displaying the claims. Note that there exists no form for data entry, saving the records. The data entry is assumed to be done by the provider from the EMR.

Data used in this prototype software is fully anonymous. Patient demographics (i.e. patient personal information) although part of data base design but not populated with records, for Protected Health Information (PHI) security reasons. Currently there is a single rule generation window, but during testing the need of multiple rule generation and association visualization windows is realized. User gives one or more initial International Classification of Disease (ICD) also known as Diagnosis Code (DxCode) and/or Current Procedure Terminology (CPT) code which are used as antecedent of all the rules. User also mentions the minimum threshold frequency and confidence criteria. Association rules are generated using Apriori-modified algorithm. Payment values (total and average charged amount, paid amount and due amount) are also calculated for all the association rules. In graphical window these association rules are displayed in the form of bar graph shown in Figure 2 and Figure 3. Each value has been made optional, by providing a check box, for displaying or not displaying in the graph. Further user can sort all the rules in ascending or descending order on the basis of any of the attribute values. Lisp version of Apriori-modified algorithm and its related functions are given below;

(defun apriori-modified(support confidence \&optional (database $*$ ex-database $*)$ items)

$\left(\mathrm{do}^{*}((\mathrm{k} 1(+\mathrm{k} 1))\right.$

(Ck (if (null items) (mapcar \#'list (all-single-intemsets database)))

(Candidates (mapcar \#'cdr Fk)))

(Fk (prune Ck support database) (prune Ck support database))

(ans nil)

)

((null Fk) (remove-redundent-items ans))

( setq ans (append ans FK))

))

(defun find-all-association-rules-modified

(\&optional (support 0.0) (confidence 0.0)

(database *ex-database*))

(generate-rules (APRIORI-MODIFIED support

confidence database) confidence

support database))

(defun generate-rules-for-given-antecedent ( $\mathrm{G}$ \&optional (minsup 0.0) (confi 0.0) (database

*ex-database*))

(let* ((SDB (remove-if \#'(lambda(a) (not (subsetp G a :test 'equalp))) database ))

(IS (all-single-intemsets SDB ))

(substs (cons nil (generate-all-subsets

(set-difference IS G :test \#'equalp))))

(Rules nil)

(dblen (length database))

(sdblen (length SDB))

(supp1 (float (/ sdblen dblen)))

)

(do ((A substs (cdr A))

(supp2 nil )

)

((null A) Rules)

(if (null (car A)) (setq supp2 (float (/ (count G

SDB :test \#'ex-equal) dblen)))

(setq supp2 (float (/ (count (car A) SDB :test

\#'subsetp) dblen))))

confi) )

(if $($ and $(>=\operatorname{supp} 2$ minsup) $(>=(/ \operatorname{supp} 2$ supp1)

(setq Rules (cons (list (/ supp2 supp1) supp2

supp1 G (car A)) Rules)))

)))

(defun Candidates(Fk)

(do ((A Fk (cdr A))

(ans nil)

)

((null A) (remove-duplicates

(remove-if \#'null ans)

:test \#'(lambda(x y)

(and (subsetp x y :test \#'equalp )

(subsetp y $\mathrm{x}$ :test \#'equalp)))))

(setq ans 
(append ans

(mapcar \#'(lambda(bb)

(if (and (equal (length (car A)) (length bb))

(some \#'(lambda $(\mathrm{x})$ (subsetp (remove x (car A)

:test 'equal) bb :test 'equal)) (car A))

(some \#'(lambda(x) (subsetp (remove x bb :test

'equal) (car A) :test 'equal)) bb)

(not (subsetp (car A) bb :test 'equal))

(not (subsetp bb (car A) :test 'equal))

)

(remove-duplicates (append (car A) bb)

:test \#'equalp) )) (cdr A))

))

)

(defun prune( $\mathrm{Ck}$ support \&optional (database

*database*))

(do ((A Ck (cdr A))

(ans nil)

)

((null A) (remove-if \#'(lambda(x)

$(<($ car $\mathrm{x})$ support $))$ ans $))$

(setq ans (cons (cons (float (/ (find-frequency (car

A) database) (length database))) (car A))

$$
\text { ans)) }
$$

))

(defun all-single-intemsets(\&optional (database *exdatabase*))

(remove-duplicates (apply \#'append database) :test \#'equal)

)

(defun find-frequency(ele \&optional (database *exdatabase*))

(count-if \#'(lambda(x)

(subsetp ele $\mathrm{x}$ :test \#'equalp) ) database) )

\section{Rule format}

Logically a rule is an implication i.e. if then_ construct. Condition part is known as antecedent and then part is known as consequent. In prototype software of this research work a rule is represented by a list of thirteen values, including antecedent and consequent. Format of the rule and its associated values is shown below;

\section{(Confidence, Support, Total_Adjusted_Amount, \\ Total_Due_Amount, \\ Total charged Amount, \\ Averäge_Due_Amount, Average_Paid_Á_moun, \\ Average_Charge,_Amount,Claim_no_List, \\ Antecedent Consequent)}

Antecedent and consequent are given at the end, are the list of ICD and/or CPT codes. Antecedent list is given by the user while consequent list is generated by Apriori-modified algorithm.

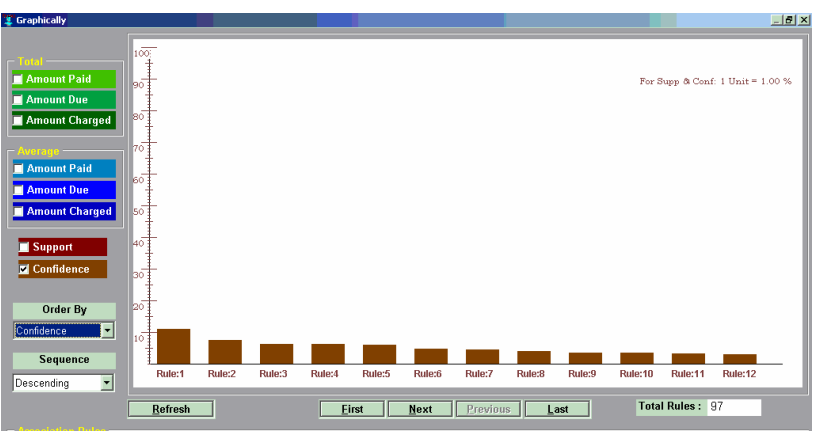

Figure 1 Graphical From Showing Confidence of Each Rule

Claim_no_List is the list of those claim numbers which are supporting this rules i.e. both antecedent and consequent codes are present in those claims. All the payments values show the payment pattern for those codes which are present as antecedent and consequent. These values are shown graphically in Figure 2 and Figure 3.

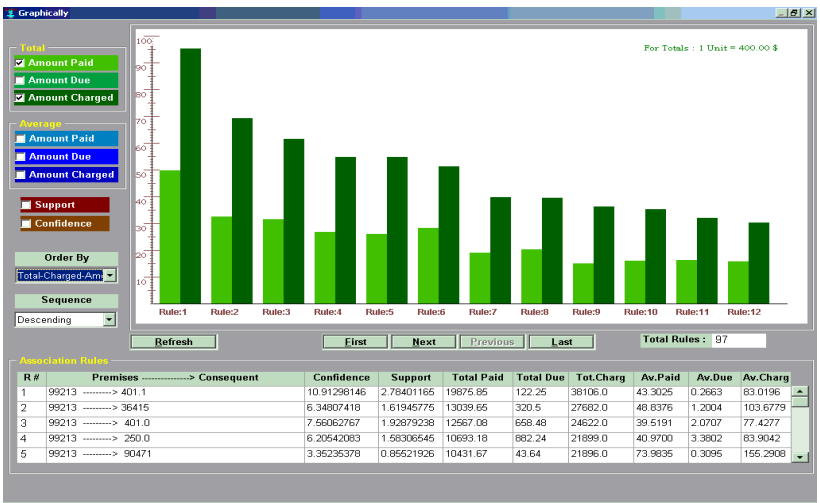

Figure 2. Graphical Form Showing Total Amounts

Support of all the generated rules is always greater than the MinSupport entered by the user. Similarly confidence, value stored as first element of rule list, is the ratio between number of claim records containing both antecedent and the consequent, with the number of records containing only antecedent. For some rules it is shown in Figure 1.

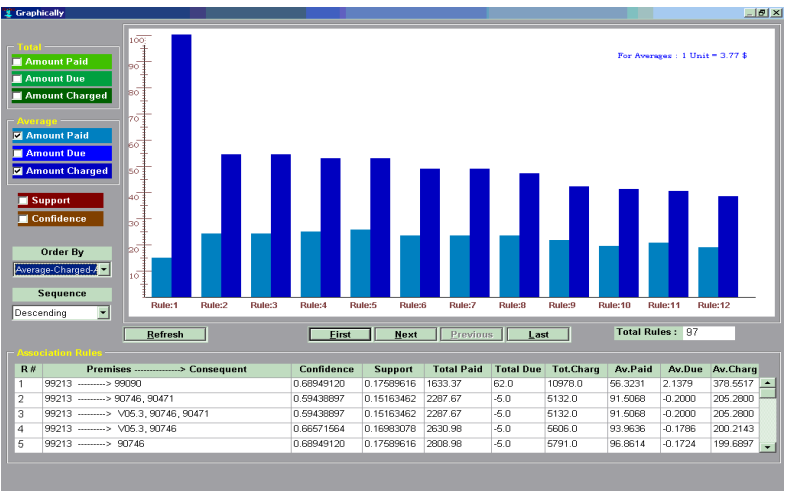

Figure 3 Graphical Form Showing Average Amounts 


\section{III.Results}

As mentioned by Agrawal [2], Apriori algorithm can be used for laying the foundations many other rule generation algorithms in future. It has been proved that Apriori algorithm is good in finding the frequent item sets of large size. But still modifications are required to adopt it to the requirements (as done in this research work).

Apriori algorithm generates Boolean association rules. In this research work payments values has been associated with each rule, hence quantitative processing can be done on the basis of any of these payment values. This is one of the possible future enhancements, which has been mentioned by Agrawal in [2], and implemented in this research.

\section{A. Discovered Characteristics of Medical Billing Data}

Data of every domain has its own characteristics; this was the basic intuition behind applying same algorithm (with some modification) in different domain. During testing following characteristics of medical billing data have been found;

\section{1) Small range of size of itemsets}

In database of retail store one transaction or itemset can has maximum of as many items as is the total number of unique items. This affects the rule generation part of Apriori algorithm which is exponentially based on the size of itemsets. This limitation is not affecting due to "small sized itemset" characteristic of billing data. An itemset (i.e. claim) consists of a minimum of two codes (one DxCode and one CPT) and maximum of ten (maximum four DxCode and six CPT). Although there is no restriction on number of CPTs applied but existing sample of data does not have more than six CPTs in one claim.

\section{2)Low frequency of individual items}

Because of the fact that a billing company accumulates data from practices of different specialties, no CPT or ICD code has large frequency. But if we take sample of data from one practice it will give relatively higher frequencies.

The maximum frequency of CPT codes is $25 \%$, with only 3 CPTs greater than $10 \%$ frequency and 26 CPTs with frequency greater than $1 \%$, while remaining CPTs (a total of 3748) have frequency less than $1 \%$. Top 5 frequent CPTs are shown in table 1 below;

TABle 1 Top 5 Frequent CPTs

\begin{tabular}{|c|c|c|c|}
\hline SR & Code & Description & Frequency \\
\hline 1 & 99213 & OFFICE/OUTPATIENT VISIT, EST & $25.511 \%$ \\
\hline 2 & 99313 & $\begin{array}{r}\text { SUBSEQ NURSING FACILITY } \\
\text { CARE - LEVEL 3 }\end{array}$ & $15.24 \%$ \\
\hline 3 & 99214 & OFFICE/OUTPATIENT VISIT, EST & $11.38 \%$ \\
\hline 4 & 99333 & $\begin{array}{r}\text { DOMICILIARY/HOME VISIT; } \\
\text { ESTAB PT-LEVEL 3 }\end{array}$ & $9.001 \%$ \\
\hline 5 & 99203 & OFFICE/OUTPATIENT VISIT, NEW & $6.38 \%$ \\
\hline
\end{tabular}

Maximum frequency of DxCodes is $15 \%$ with only 3 DxCodes greater than $10 \%$. There are 56 DxCodes having frequency greater than $1 \%$. Top 5 frequent DxCodes are shown in table 2 given below.

\begin{tabular}{|c|c|c|c|}
\multicolumn{5}{c}{ TABLE 2. TOP 5 Frequent DxCODES } \\
\hline SR & DxCode & Description & Frequency \\
\hline 1 & 401.0 & $\begin{array}{c}\text { MALIGNANT ESSENTIAL } \\
\text { HYPERTENSION }\end{array}$ & $15.18 \%$ \\
\hline 2 & 250.0 & $\begin{array}{c}\text { DIABETES MELLITUS WITHOUT } \\
\text { COMPLICATION TYPE II OR } \\
\text { UNSPECIFIED TYPE NOT } \\
\text { STATED AS UNCONTROLLED }\end{array}$ & $11.50 \%$ \\
\hline 3 & 401.1 & $\begin{array}{c}\text { BENIGN ESSENTIAL } \\
\text { HYPERTENSION }\end{array}$ & $10.50 \%$ \\
\hline 4 & 428.0 & $\begin{array}{c}\text { CONGESTIVE HEART FAILURE } \\
\text { UNSPECIFIED }\end{array}$ & $5.43 \%$ \\
\hline 5 & 272.0 & $\begin{array}{c}\text { PURE } \\
\text { HYPERCHOLESTEROLEMIA }\end{array}$ & $5.00 \%$ \\
\hline
\end{tabular}

Note that in rule generation process only those rules are selected in which combined frequency of antecedent and consequent is greater than the threshold frequency given by user. If we use most frequent DxCode as antecedent and most frequent CPT as consequent, their combined frequency is 1.93 $\%$ which is lower than the expected value.

The highest combined frequency is $3.29 \%$ which is for DxCode 401.0 and 250.0. While the combined frequency of most frequent CPT code (i.e. 99213) is $2.784 \%$, which is with DxCode 401.1. Thus we don't expect any rule with frequency (support) higher than $5 \%$.

\section{3) Regular patterns in payment values}

The prototype software developed for this research work does not only finds the associations between DxCodes and CPTs but also shows us payments patterns against those associations. Payment record has four values, first one Charged Amount, it is the amount charged, or claimed by the provider in a patient claim, it is always highest among other payment values, second one is the adjusted amount it is that part of charged amount which is removed, rejected, or not paid by any of the payers. Third value is the paid amount, which refer to that amount which is paid by any of the payers (normally primary insurance of a patient). Fourth value is due amount, which represent the pending amount of a claim. Initially all the charged amount is due amount. But mostly after payment from primary insurance, some part of charged amount is left as due amount, which is later on paid by secondary insurance or the patient himself/herself.

During the result analysis phase, it is revealed that charged amount has relatively less consistent pattern. On the other hand paid amount appears to be more systematic and having consistent patterns. That might be due to the reason that any insurance carefully analyze, evaluate, and process the claims before making a payment.

\section{B. Empty Rule Generation}

Rule generation part of Apriori algorithm has been modified to generate empty rules. In an empty rule consequent part of the rule is empty or nil, and the antecedent part provided by the user. A greater confidence value of such a rule tells the medical service provider not to add any further code to the claim. 


\section{IV.Future Possibilities}

There are a lot of possibilities and room for future research work. Some of them are mentioned below.

\section{A. Multiple Rule Generation}

Current prototype software has one rule generation window, but during the testing it was realized that it would be better to have multiple rule visualization windows for one algorithm and multiple rule generation algorithms can be implemented. This will give us a comparative view of outcome and the performance of rule generation algorithms.

\section{B. Diagnostic Decision Support}

Decision support in diagnosing process is an old research area, but up till now it has been constrained by the dependency on human medical experts for the population of knowledge in the system. Now data mining features embedded in such systems, will add up another dimension view in diagnostic decision making process.

\section{Distributed Data Mining}

At billing company data from all practices is collected. A provider can perform data mining operations on its own practice side by using the EMR. This can be extended to the data mining operations on the data of the billing company, a global database. Results of the data mining operations on the global database can be sent, compared and utilized with the data mining operations on local database of a single practice.

\section{V.Conclusion}

After having all the results and trying different approaches, it is found that it is a priori property (i.e. a frequent set can only be generated from frequent subsets) which makes Apriori algorithm good for finding frequent itemsets from billing database. Although some modifications have been made in the algorithm to cope with the requirement, but basic rule generation process is same as that of Apriori algorithm.

It is found that use of high level language can overcome the requirement of lexical sorting of transaction records, which was one weak point of original implementation of Apriori algorithm. Further the use of higher level language like lisp, can allow us to associate some quantities (like payments) with association rules and thus perform quantitative analysis in rule generation process. But these higher language features may be costing some response time.

There is a margin of improvement in rule generation part of Apriori algorithm as currently it is exponentially based on the size of itemsets. This weakness is not affecting the performance of the prototype software developed for this research due to the "small itemset size" characteristic of billing database.

Decision support module, based on Apriori-modified algorithm, is useful for increasing the consistency of charged amount thus can improve payments. And due to less processing time on insurance side it will reduce aging of claims.

\section{Acknowledgements}

Many thanks to Dr. Mohammad Jamil Sawar and Mr. Mahmud Haq for their encouragement and firm support

\section{References}

[1] R. Agrawal, T. Imielinski, and A. N. Swami. "Mining association rules between sets of items in large databases." In Peter Buneman and Sushil Jajodia, editors, Proceedings of the 1993 ACM SIGMOD Intl. Conference on Management of Data, pages 207-216, Washington, D.C., 26-28 1993

[2] R. Agrawal and R. Srikant. "Fast algorithms for mining association rules." In Jorge B. Bocca, Matthias Jarke, and Carlo Zaniolo, editors, Proceedings of 20th Intl. Conference of Very Large Data Bases, VLDB, pages 487-499. Morgan Kaufmann, 12-15 1994.

[3] "Healthcare Informatics, 100 - IT Companies by Healthcare Revenue" Healthcare Informatics, June 2004 